\title{
A New Approach to the Magnetochemical Theory Near Death Experiences and of Formation Phosphine from Phosphates
}

\author{
Aibassov Erkin Zhakenovich ${ }^{1}$, Nakisbekov Narymzhan ${ }^{1}$, Yemelyanova Valentina ${ }^{1}$ and Savizky Ruben ${ }^{2}$ \\ 1. Research Institute of New Chemical Technologies and Materials, Kazakh National University Al-Farabi, Almaty 005012, \\ Kazakhstan \\ 2. Columbia University, 3000 Broadway, New York, NY, 10027, USA
}

\begin{abstract}
The authors have proposed a new approach to the theory of formation of phosphine from phosphates and death. According to this hypothesis, the initial impetus for the beginning of the dying process is the formation of phosphates and phosphine decomposition of adenosine triphosphate molecule. This process makes people survived clinical death vision of "blue and white tunnel" and the separation of the soul from the body. The authors proposed chemical mechanism for the recovery of phosphate to phosphine.
\end{abstract}

Key words: Magnetic field, phosphine, adenosine triphosphate, theory of death, "blue and white tunnel"

\section{Introduction}

Death-termination, is a full stop of biological and physiological processes of the body's vital functions. The phenomena, often cause death-aging, malnutrition, disease and accidents. Shortly after the death, the body of living organisms begin to decompose.

The authors first time offered to consider the effect of the magnetic field and chemical reactions to the recovery of phosphates and phosphine free radicals, which lead to an acceleration of the process of dying. The authors were interested to see what possible chemical reactions and electromagnetic processes are in the event of near-death state. What is the reason of phosphenes? What chemical reactions may cause the formation of "white and blue tunnel (light)"?

\section{Theory}

It is well known that most people who have experienced clinical death told us that they had seen

Corresponding author: Aibassov Erkin Zhakenovich, professor, research field: metal organic chemistry of uranium and thorium, $\mathrm{As}, \mathrm{Sb}$ and $\mathrm{Bi}$. "white and blue tunnel (light)" [1-10]. The human retina contains about 120 million rods. This highly specialized cells that convert light stimuli into nervous excitement. Rods are sensitive to light due to the presence in them of a specific pigment-rhodopsin. Under the influence of light is a series of very fast reactions and discoloration of the visual pigment. The sensitivity of the rods is sufficient to register a hit even 2-3 photons.

There is an ongoing outward potassium current through nongated $\mathrm{K}^{+}$-selective channels. This outward current tends to hyperpolarize the photoreceptor at around $-70 \mathrm{mV}$ (the equilibrium potential for $\mathrm{K}^{+}$). There is also an inward sodium current carried by cGMP-gated sodium channels. This so-called "dark current" depolarizes the cell to around $-40 \mathrm{mV}$. Note that this is significantly more depolarized than most other neurons. A high density of $\mathrm{Na}^{+}-\mathrm{K}^{+}$pumps enables the photoreceptor to maintain a steady intracellular concentration of $\mathrm{Na}^{+}$and $\mathrm{K}^{+}$. The human eye is sensitive to electromagnetic radiation in the wavelength range 400-750 $\mathrm{nm}$ (visible light). 
Phosphenes can be directly induced by mechanical, electrical, or magnetic stimulation of the retina or visual cortex as well as by random firing of cells in the visual system. Phosphenes have also been reported by meditators (commonly called nimitta), people who go for long periods without visual stimulation (also known as the prisoner's cinema), or those who are using psychedelic drugs.

Phosphenes have also been created by intense, changing magnetic fields, such as with transcranial magnetic stimulation. These fields can be positioned on different parts of the head to stimulate cells in different parts of the visual system. They also can be induced by alternating currents that entrain neural oscillation as with transcranial alternating current stimulation. In this case they appear in the peripheral visual field. This claim has been disputed; the alternative hypothesis is that current spread from the occipital electrode evokes phosphenes in the retina. Phosphenes created by magnetic fields are known as magnetophosphenes.

Thus, the authors assume that when the dying situations occur in the brain chemical processes to recover phosphates phosphine that's just leads to the observed by many people who have experienced clinical death "white or blue tunnel".

\section{Results and Discussion}

During NDEs (near death experiences), the subjects' heartbeat and breathing are temporarily suspended, and they exhibit flattened brain waves in the EEG (electroencephalogram) and absence of auditory evoked potentials from the brainstem. NDEs consist of some frequent components such as OBE (out-of-body experience) (separation of consciousness from the physical body), passage through a dark tunnel, encounters with bright lights, meeting deceased relatives.

Ultraweak (bio) chemiluminescence spectral intensity peaks were observed at 480, 520-530, 570, $620-640$ and $680-700 \mathrm{~nm}$.
According to Hameroff and Chopra, the observed gamma oscillations reported in the studies can be linked to consciousness states that involve particularly low-energy quantum entanglements that persisted over time while other brain functions have run out of energy supply. Consciousness could persist outside the physical body but remains localized on the level of Planck-scale geometry. "A quantum basis for consciousness also raises the scientific possibility of an afterlife, of an actual soul leaving the body and persisting as entangled fluctuations in quantum spacetime geometry" (Hameroff and Chopra, 2010). When the patient's physical body is resuscitated, the quantum information can reenter it, and the subjects may be able to recall their experience involving NDEs.

Our thinking processes and indeed every decision made at a given moment can be a coherent and convergent dynamic (discrete events) manifestation of our unconscious cognitive (essential picture-representation) processes due to the billions of non-conscious processes.

The idea by Hameroff and Chopra that the self-consciousness could continue outside the body but remains at a level of Planck-scale geometry (Planck length is about $10^{-33} \mathrm{~cm}$ ), may be realized by potential quantum-like properties of biophotons (and by virtual photon particles). The authors hypothesize that human self-consciousness is an individual-specific abstract manifestation of language and experience-dependent expression of conscious plus unconscious exposure and experiences of an individual.

It is known that light is produced by closing an electrical circuit. This process can be described by Eq. (1):

$$
\Delta \mathrm{E} / \Delta \mathrm{B}=\mathrm{c}
$$

where, $\Delta \mathrm{E}$ is an electric field, $\Delta \mathrm{B}$ a magnetic field, and c-velocity of light, $300 \mathrm{~km} / \mathrm{sec}$.

It is known that in the oxidation of elemental phosphorus or phosphine with oxygen in the air is a "white or blue glow". This process is described by the Eq. (2): 


\section{A New Approach to the Magnetochemical Theory near Death Experiences and of Formation Phosphine from Phosphates}

$$
\mathrm{P}(+5)+8 \mathrm{H}^{+} \rightarrow \mathrm{P}(-3)
$$

The authors believe that the near-death process chemical reactions occur restore pentavalent phosphorus (ATP) to phosphine (-3).

\section{Conclusions}

The authors proposed a novel biophysical concept regarding the appearance of brilliant lights during NDEs (near death experiences). Perceiving brilliant light in NDEs has been proposed to arise due to the reperfusion that produces unregulated overproduction of free radicals and energetically excited molecules that can generate a transient enhancement of bioluminescent biophotons in different areas of the brain, including retinotopic visual areas. If this excess of bioluminescent photon emission exceeds a threshold in retinotopic visual areas, this can appear as (phosphene) lights because the brain interprets these intrinsic retinotopic bioluminescent photons as if they originated from the external physical world. The authors briefly describe our biophysical visual representation model that may explain brilliant lights experienced during NDEs (by phosphenes as biophotons) and REM sleep associated dream-like intrinsic visual imageries through biophotons in NDEs. The authors link our biophysical visual representation notion to self-consciousness that may involve extremely low-energy quantum entanglements.

Here the authors presented some novel ideas to stimulate new concepts that may facilitate the understanding of the phenomena of NDEs.

Hameroff and Chopra (2010) speculated that self-consciousness involves extremely low-energy quantum entanglements that could return to the material body if the person is resuscitated. The authors linked our biophysical visual representation notion to self-consciousness that may involve extremely low-energy quantum entanglements by means of biophotons.

\section{References}

[1] Agrillo, C. 2011. "Near-death Experience: Out-of-Body and Out-of-Brain." Rev. Gen. Psychol. 15: 1-10.

[2] Appelby, L. 1989. "Near-death Experience: Analogous to Other Stress Induced Physiological Phenomena." $B M J$ 298: 976-7.

[3] Blackmore, S. J. 1996. "Near-death Experiences." J. $R$. Soc. Med. 89: 73-6.

[4] Blackmore, S. J., and Troscianko, T. 1988. "The Physiology of the Tunnel." J. Near Death Stud. 8: 15-28.

[5] Bókkon, I. 2008. "Phosphene Phenomenon: A New Concept." Biosystems 92: 168-74.

[6] Bonilla, E. 2011. "Near-death Experiences." Invest. Clin. 52: 69-99.

[7] Chang, J. J. 2008. "Physical Properties of Biophotons and Their Biological Functions." Indian J. Exp. Biol. 46: 371-7.

[8] French, C. C. 2001. "Dying to Know the Truth: Visions of a Dying Brain, or False Memories." Lancet 358: 2010.

[9] Bókkon, I., Mallick, B., and Tuszynski, J. 2013. "Near Death Experiences: A Multidisciplinary Hypothesis." Front Hum Neurosci. 7: 533.

[10] French, C. C. 2005. "Near-death Experiences in Cardiac Arrest Survivors." Prog. Brain Res. 150: 351-67. 